\title{
Burden of vision loss in the Eastern Mediterranean region, 1990-2015: findings from the Global Burden of Disease 2015 study
}

\author{
GBD 2015 Eastern Mediterranean Region Vision Loss Collaborators ${ }^{1}$
}

Received: 2 May 2017/Revised: 22 June 2017 / Accepted: 23 June 2017/Published online: 3 August 2017

(c) The Author(s) 2017. This article is an open access publication

\begin{abstract}
Objectives To report the estimated trend in prevalence and years lived with disability (YLDs) due to vision loss (VL) in the Eastern Mediterranean region (EMR) from 1990 to 2015. Methods The estimated trends in age-standardized prevalence and the YLDs rate due to VL in 22 EMR countries were extracted from the Global Burden of Disease (GBD) 2015 study. The association of Socio-demographic Index (SDI) with changes in prevalence and YLDs of VL was evaluated using a multilevel mixed model.

Results The age-standardized prevalence of VL in the EMR was $18.2 \%$ in 1990 and $15.5 \%$ in 2015. The total agestandardized YLDs rate attributed to all-cause VL in EMR was 536.9 per 100,000 population in 1990 and 482.3 per 100,000 population in 2015 . For each 0.1 unit increase in SDI, the age-standardized prevalence and YLDs rate of VL showed a reduction of $1.5 \%(p<0.001)$ and 23.9 per 100,000 population $(p<0.001)$, respectively.
\end{abstract}

This article is part of the supplement "The state of health in the Eastern Mediterranean Region, 1990-2015."

The members of GBD (Global Burden of Disease) 2015 Eastern Mediterranean Region Vision Loss Collaborators are listed at the end of the article. Ali H. Mokdad, on behalf of GBD 2015 Eastern Mediterranean Region Vision Loss Collaborators, is the corresponding author.

Electronic supplementary material The online version of this article (doi:10.1007/s00038-017-1000-7) contains supplementary material, which is available to authorized users.

GBD 2015 Eastern Mediterranean Region Vision Loss Collaborators

mokdaa@uw.edu

1 Institute for Health Metrics and Evaluation, University of Washington, Seattle, WA, USA
Conclusions The burden of VL is high in the EMR; however, it shows a descending trend over the past 25 years. EMR countries need to establish comprehensive eye care programs in their health care systems.

Keywords Eastern Mediterranean region - Global burden of disease · Vision impairment · Vision disorder

\section{Introduction}

Vision loss is an important public health issue worldwide. About $90 \%$ of people with visual impairment live in developing countries (Congdon et al. 2003; Tabbara 2001; WHO 2004, 2013). According to the Global Burden of Disease (GBD) 2015 study, 34.3 million people are blind globally. In addition, 214 million and 24.3 million people suffer from moderate and severe vision impairment (VI), respectively. Vision loss was the third-ranked impairment after anemia and hearing loss in the GBD 2015 study (GBD 2015). Vision loss affects the quality of life of the affected individuals and their families, increases the risk of death by raising the risk of accidents, and increases the financial burden (McCarty et al. 2001; Taylor et al. 1991, 2006). The World Health Organization (WHO) Global Action Plan 2014-2019 emphasized the importance of collecting data on the burden and causes of VI. Periodic studies were recommended to identify the burden of vision loss and the avoidable causes of VI and blindness to achieve a Global Action Plan target and plan health policies (WHO 2013).

Uncorrected refractive errors (RE), cataract, glaucoma, age-related macular degeneration (AMD), diabetic retinopathy (DR), trachoma, and corneal opacities were the main causes of global VI reported by WHO in 2010 
(Pascolini and Mariotti 2012). Uncorrected RE, cataract, and glaucoma accounted for 43, 33, and $2 \%$ of VI, respectively (Pascolini and Mariotti 2012). Hence, almost $80 \%$ of VI is preventable or treatable, and cost-effective interventions can decrease the burden of VI (WHO 2013).

The Eastern Mediterranean region (EMR) has a population of about 583 million and consists of 22 countries (WHO 2017). These countries are not uniform in terms of lifestyle, gross domestic product, and socioeconomic status (Mandil et al. 2013; Mokdad et al. 2014). A previous study reported a descending trend in age-standardized prevalence of blindness (from 2.1 to $1.1 \%$ ) and moderate and severe VI (from 7.1 to $4.5 \%$ ) in this region in 2010 (Khairallah et al. 2014). Despite that, the EMR is one of four regions with a greater than $4 \%$ prevalence of blindness among older adults ( $\geq 50$ years), compared to $\leq 0.4 \%$ in high-income regions (Stevens et al. 2013). The current study aims to present trends in prevalence and years lived with disability (YLDs) rate for the main causes of vision loss in EMR countries by sex and age from 1990 to 2015 using the results of the GBD 2015 study. Considering the diversity of the EMR countries in terms of socioeconomic status, the relationship between the prevalence of vision loss and Socio-demographic Index (SDI) was also evaluated.

\section{Methods}

We used data from the Global Burden of Disease 2015 study (GBD 2015). The methodology of the GBD 2015 study for estimating the prevalence of vision loss has been comprehensively described in a recent GBD publication (GBD 2015). In brief, the GBD group estimated the prevalence of vision loss by defining VI and blindness, providing the input model, and defining the modeling strategy. VI was defined as visual acuity (VA) $<6 / 18$ based on the Snellen chart, while the blindness definition was $\mathrm{VA}<3 / 60$ or visual field around central fixation $<10 \%$. Uncorrected RE, cataract, glaucoma, macular degeneration, and other causes including DR, trachoma, vitamin A deficiency, retinopathy of prematurity (ROP), meningitis, encephalitis, and onchocerciasis were used for modeling vision loss (GBD 2015).

The vision loss data were from population-based studies that measured VA. Both peer-reviewed publications and gray literature were used. Those which reported the causes of vision loss were used for estimating the VI and blindness prevalence due to cataract, glaucoma, macular degeneration, DR, and other causes (GBD 2015). Studies missing bestcorrected or presenting VA were excluded (GBD 2015).

A systematic literature review was performed for the period of January 1, 2013-May 20, 2015, to add new evidence to that compiled for GBD 2013. Additionally, the data were extracted from WHO-sponsored Studies on
Global Ageing and Adult Health (SAGE) and the United States National Health and Examination Surveys (NHANES) as the nationally representative reviews that measured VA. In addition to SAGE and NHANES, the Surveys of Health, Ageing, and Retirement in Europe (SHARE); the Multi-Country Survey Study on Health and Responsiveness (MCSS); and the World Health Surveys (WHS) studies were assessed to extract the data with selfreported near VA (GBD 2015).

The prevalence of vision loss was modeled in three stages. At first, the prevalence of moderate and severe VI, blindness, and presbyopia was evaluated to calculate the total presenting vision loss estimation. Secondly, the proportion of presenting vision loss attributed to uncorrected RE was estimated. Thirdly, the prevalence of vision loss due to cataract, glaucoma, macular degeneration, DR, ROP, trachoma, vitamin A deficiency, onchocerciasis, meningitis, and other causes was assessed (GBD 2015).

The YLDs rate, a GBD metric, demonstrates years lived in less than ideal health and is calculated by sequela as prevalence multiplied by the disability weight for the condition associated with that sequela (GBD 2015).

We extracted the estimated trends in prevalence and YLDs rates for vision loss and four leading causes, including refraction and accommodation disorders, cataract, glaucoma, and macular degeneration, in Afghanistan, Bahrain, Djibouti, Egypt, Iran, Iraq, Jordan, Kuwait, Lebanon, Libya, Morocco, Oman, Pakistan, Palestine, Qatar, Saudi Arabia, Somalia, Sudan, Syria, Tunisia, the United Arab Emirates, and Yemen from 1990 to 2015 from the GBD 2015 study using an online interactive tool (https://vizhub.healthdata.org/gbd-compare/) developed by the Institute for Health Metrics and Evaluation. We also determined the relationship between SDI and the reduction in prevalence of vision loss and YLDs rate. The SDI was developed for GBD 2015 to provide an interpretable synthesis of overall development, as measured by lag-dependent income per capita, average educational attainment in the population over 15 years, and the total fertility rates. In GBD 2015, SDI was computed by rescaling each component to the scale of zero to one, and then taking the geometric mean of these values for each location-year. Zero indicates the lowest observed educational attainment, lowest income per capita, and highest fertility rate from 1980 to 2015, and one indicates the highest observed educational attainment, highest income per capita, and lowest fertility rate during that time (GBD 2015).

\section{Statistical methods}

A multilevel linear model was used to assess the relation of SDI values extracted from the GBD 2015 study for 22 countries in the EMR and the changes in prevalence and 
YLDs during the period 1990-2015. In this way, the probable geographic correlation was also considered. All statistics were presented with $95 \%$ uncertainty intervals (95\% UI). Statistical analysis was performed using lme4 package in R software (version 3.2.3) (Bates et al. 2015).

\section{Results}

The age-standardized prevalence of vision loss in the EMR was $18.2 \%$ (95\% UI $17.5-19 \%$ ) in 1990 and $15.5 \%$ (95\% UI 14.8-16.2\%) in 2015. The total age-standardized YLDs rate attributed to vision loss in EMR was 536.9 per 100,000 population (95\% UI 378.5-746) in 1990 and 482.3 per 100,000 population (95\% UI 342.5-667.8) in 2015. Vision loss was more prevalent in females than males in both 1990 and $2015(p<0.001)$. There were similar findings in terms of the estimated YLDs rate $(p<0.001)$ (Fig. 1).

Table 1 shows decreased prevalence of vision loss and the attributed YLDs rate in all 22 countries from 1990 to 2015. The highest decrease was observed in Oman (prevalence -26.6, YLDs -16.2), while the lowest was observed in Somalia (prevalence -1.6, YLDs -2.0).

A descending trend in the age-standardized prevalence and YLDs rates for vision loss was noted among both sexes from 1990 to 2015 (Fig. 2).
The age-standardized prevalence and YLDs rates of vision loss in the EMR were higher than the global rate and ranked third following the Southeast Asia and Africa regions in 1990 and 2015. However, this region had the highest reduction from 1990 to 2015 compared to all six world regions (ESM_1).

\section{Age-standardized prevalence and YLDs for causes of vision loss}

\section{Refraction and accommodation disorders}

The total age-standardized prevalence of refraction and accommodation disorders in the EMR was $12.9 \%$ (95\% UI $12.4-13.4 \%$ ) in males and $17.8 \%$ (95\% UI $17.1-18.6 \%$ ) in females in 1990. In 2015, these values were $10.8 \%$ (95\% UI $10.3-11.2 \%$ ) in males and $14.8 \%$ (95\% UI $14.1-15.5 \%)$ in females. The prevalence of this disorder in 1990 was highest in Yemen and lowest in Lebanon. In 2015, Afghanistan had the highest prevalence in terms of refraction and accommodation disorders (ESM_2). The age-standardized YLD rate from refraction and accommodation disorders in the EMR in 1990 amounted to 263.3 (95\% UI 174.3-395.6) per 100,000 males and 337.9 (95\% UI 221.9-518.7) per 100,000 females. In 2015, YLDs attributed to refraction and accommodation disorders in the EMR were 231.9 (95\% UI
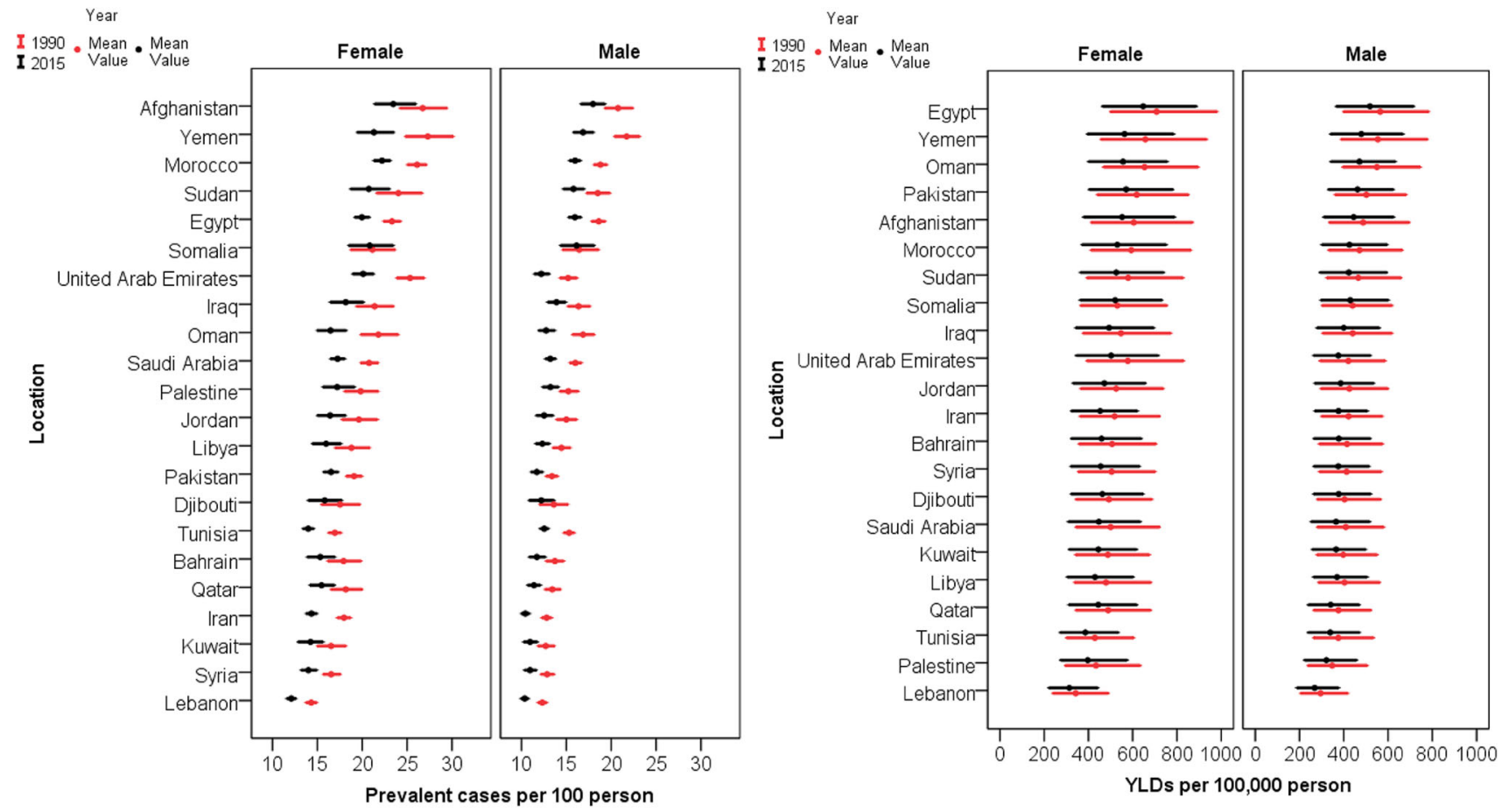

Fig. 1 Age-standardized prevalence and years lived with disability (YLDs) rate of vision loss in Eastern Mediterranean Region countries in 1990 and 2015 (Global Burden of Disease Study 2015, Eastern Mediterranean Countries, 1990-2015) 
Table 1 Age-standardized prevalence and years lived with disability rate per 100,000 population of vision loss in the Eastern Mediterranean Region in 1990 and 2015 (Global Burden of Disease Study 2015, Eastern Mediterranean Countries, 1990-2015)

\begin{tabular}{|c|c|c|c|c|c|c|c|c|c|c|c|c|c|c|}
\hline \multirow[t]{4}{*}{ Location } & \multicolumn{6}{|c|}{ Prevalence $(\%)$} & \multirow{4}{*}{$\begin{array}{l}\text { Percent } \\
\text { change }\end{array}$} & \multicolumn{6}{|c|}{ YLDs per 100,000 population } & \multirow{4}{*}{$\begin{array}{l}\text { Percent } \\
\text { change }\end{array}$} \\
\hline & \multicolumn{3}{|l|}{1990} & \multicolumn{3}{|l|}{2015} & & \multicolumn{3}{|l|}{1990} & \multicolumn{3}{|l|}{2015} & \\
\hline & \multirow[t]{2}{*}{ Value } & \multicolumn{2}{|c|}{$95 \% \mathrm{UI}$} & \multirow[t]{2}{*}{ Value } & \multicolumn{2}{|c|}{$95 \%$ UI } & & \multirow[t]{2}{*}{ Value } & \multicolumn{2}{|c|}{$95 \% \mathrm{UI}$} & \multirow[t]{2}{*}{ Value } & \multicolumn{2}{|c|}{$95 \% \mathrm{UI}$} & \\
\hline & & Lower & Upper & & Lower & Upper & & & Lower & Upper & & Lower & Upper & \\
\hline $\begin{array}{l}\text { Eastern } \\
\text { Mediterranean } \\
\text { Region }\end{array}$ & 18.2 & 17.5 & 19 & 15.5 & 14.8 & 16.2 & -15.1 & 536.9 & 378.5 & 746 & 482.3 & 342.5 & 667.8 & -10.2 \\
\hline Afghanistan & 23.8 & 21.9 & 25.8 & 20.7 & 19.1 & 22.4 & -12.9 & 547.1 & 374.5 & 785.5 & 499 & 341.9 & 711.6 & -8.8 \\
\hline Bahrain & 15.5 & 14.3 & 16.9 & 13.2 & 12.2 & 14.4 & -14.7 & 454.2 & 318.6 & 631.1 & 412.6 & 290.3 & 573.3 & -9.2 \\
\hline Djibouti & 15.6 & 13.9 & 17.5 & 14.1 & 12.5 & 15.7 & -10.2 & 450.2 & 312.3 & 627.6 & 421.1 & 291.2 & 587.5 & -6.5 \\
\hline Egypt & 21.1 & 20.3 & 21.9 & 18.1 & 17.3 & 18.8 & -14.5 & 642.7 & 456.6 & 893.5 & 586.8 & 418.2 & 807.3 & -8.7 \\
\hline Iran & 15.3 & 14.7 & 15.9 & 12.3 & 11.8 & 12.9 & -19.5 & 468.3 & 331.2 & 647 & 413.2 & 295.4 & 563.9 & -11.8 \\
\hline Iraq & 18.9 & 17.4 & 20.6 & 16.1 & 14.8 & 17.6 & -14.7 & 495.3 & 339.9 & 697 & 449.2 & 312.2 & 636.2 & -9.3 \\
\hline Jordan & 17.3 & 15.9 & 18.7 & 14.5 & 13.3 & 15.8 & -16.1 & 473.9 & 329.1 & 667.6 & 429 & 299.7 & 596 & -9.5 \\
\hline Kuwait & 14.3 & 13.3 & 15.5 & 12.4 & 11.4 & 13.4 & -13.7 & 436.2 & 305.6 & 604.9 & 398.8 & 280.1 & 552.1 & -8.6 \\
\hline Lebanon & 13.3 & 12.8 & 13.9 & 11.2 & 10.7 & 11.6 & -16.1 & 319.4 & 220.9 & 457.4 & 290.6 & 202.7 & 408.5 & -9 \\
\hline Libya & 16.5 & 15.3 & 18 & 14.2 & 13.2 & 15.4 & -14 & 439.8 & 310.3 & 613.3 & 400.3 & 283.8 & 556.6 & -9 \\
\hline Morocco & 22.7 & 21.9 & 23.5 & 19.2 & 18.5 & 19.9 & -15.3 & 536.5 & 373.2 & 770.7 & 480.1 & 335.1 & 677.7 & -10.5 \\
\hline Oman & 19.3 & 17.9 & 20.8 & 14.1 & 13.2 & 15.3 & -26.6 & 602.4 & 434.9 & 824.1 & 505 & 364.4 & 683.1 & -16.2 \\
\hline Pakistan & 16 & 15.3 & 16.8 & 14.1 & 13.4 & 14.8 & -12.3 & 555.3 & 395.6 & 760.1 & 514.7 & 368.2 & 699 & -7.3 \\
\hline Palestine & 17.6 & 16.3 & 19 & 15.3 & 14.1 & 16.5 & -13.3 & 392.7 & 267.6 & 574.1 & 360.4 & 249.3 & 517.5 & -8.2 \\
\hline Qatar & 15 & 14 & 16.1 & 12.6 & 11.8 & 13.5 & -15.7 & 414.5 & 290.7 & 577.6 & 373.3 & 261.2 & 515.5 & -9.9 \\
\hline Saudi Arabia & 18.2 & 17.5 & 18.9 & 15 & 14.4 & 15.7 & -17.4 & 451.8 & 310.1 & 644.2 & 402.3 & 275.8 & 569.2 & -11 \\
\hline Somalia & 18.9 & 16.8 & 21.2 & 18.6 & 16.6 & 20.8 & -1.6 & 487.1 & 335.3 & 690.9 & 477.2 & 329.9 & 671 & -2 \\
\hline Sudan & 21.3 & 19.7 & 23.1 & 18.3 & 16.8 & 20 & -14.1 & 524.5 & 360.6 & 744.2 & 476.1 & 327.7 & 669.7 & -9.2 \\
\hline Syria & 14.7 & 13.9 & 15.6 & 12.5 & 11.8 & 13.4 & -14.7 & 458.8 & 321.9 & 637.8 & 416.7 & 294.7 & 574.1 & -9.2 \\
\hline Tunisia & 16.1 & 15.5 & 16.7 & 13.3 & 12.8 & 13.8 & -17.6 & 402.3 & 280.7 & 571.8 & 364 & 254.4 & 506 & -9.5 \\
\hline $\begin{array}{c}\text { United Arab } \\
\text { Emirates }\end{array}$ & 18.8 & 17.7 & 19.9 & 14.2 & 13.4 & 15.2 & -24.4 & 478.5 & 329 & 678.5 & 408.6 & 284.8 & 572.7 & -14.6 \\
\hline Yemen & 24.8 & 23 & 26.8 & 19.2 & 17.8 & 20.7 & -22.8 & 611.7 & 425.3 & 870.5 & 522.5 & 367.4 & 726.7 & -14.6 \\
\hline
\end{tabular}

$U I$ uncertainty interval, YLDs years lived with disability

154.4-344.7) per 100,000 males and 296.4 (95\% UI 195.4-446.4) per 100,000 females. In 1990 and 2015, Egypt had the highest age-standardized YLDs per 100,000 person, and Lebanon had the lowest (ESM_2).

\section{Cataract}

The age-standardized prevalence of cataract in the EMR was $1.5 \%$ (95\% UI 1.3-1.6\%) among males and $1.8 \%$ (95\% UI 1.6-2.0\%) among females in 1990. The corresponding values were $1.3 \%$ (95\% UI 1.2-1.5\%) in males and $1.7 \%$ (95\% UI 1.5-1.9\%) in females in 2015 (ESM_3). Cataract was most prevalent in Pakistan and least prevalent in Libya in both 1990 and 2015.
Age-standardized YLDs attributed to cataract in the EMR in 1990 were 97.9 (95\% UI 70.3-132.7) per 100,000 population in males and 124.4 (95\% UI 89.0-168.3) per 100,000 population in females. In 2015 , the corresponding values were 84.9 (95\% UI 60.6-113.9) per 100,000 population in males and 113.6 (95\% UI 81.6-152.5) per 100,000 population in females (ESM_3). The highest rate of YLDs per 100,000 population for cataract were observed in Pakistan, and the lowest in Lebanon both in 1990 and 2015.

\section{Glaucoma}

In both 1990 and 2015, the highest age-standardized prevalence of glaucoma was observed in Egypt, and the 

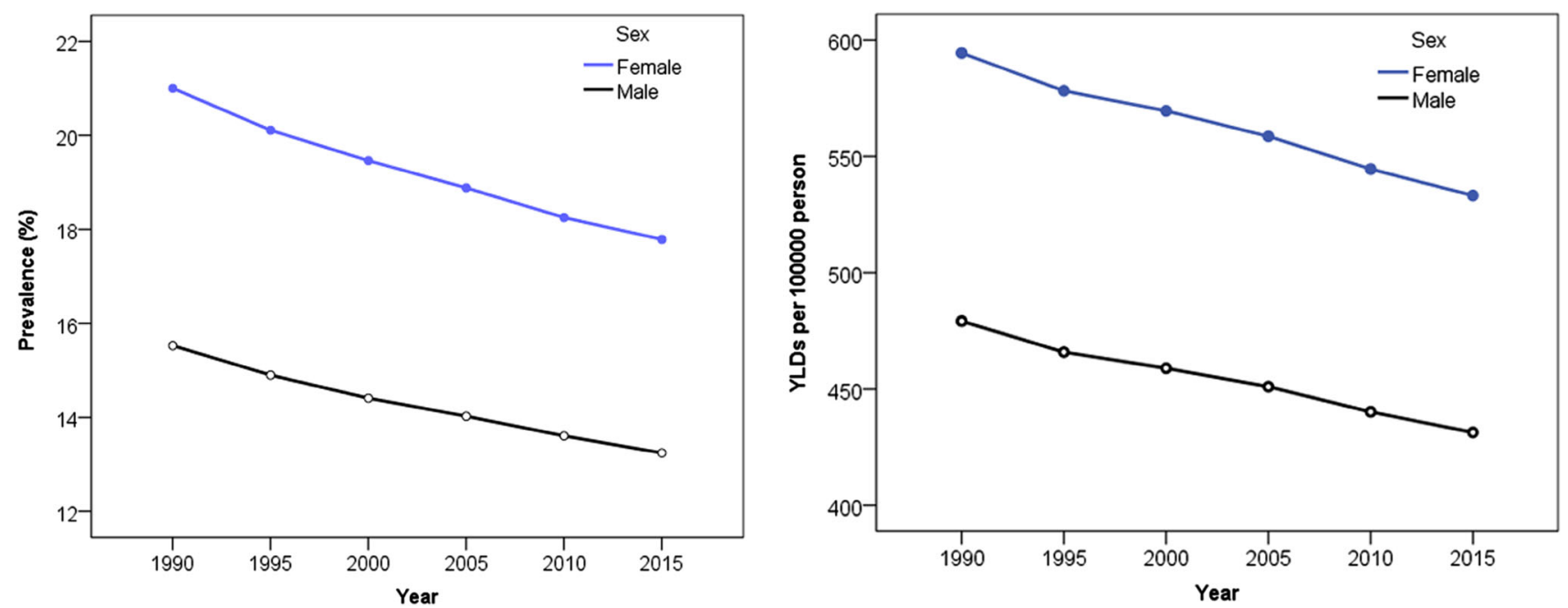

Fig. 2 Trends in age-standardized prevalence and years lived with disability (YLDs) rate of vision loss by gender in the Eastern Mediterranean Region from 1990 to 2015 (Global Burden of Disease Study 2015, Eastern Mediterranean Region, 1990-2015)

lowest was reported in Afghanistan. Total age-standardized prevalence of glaucoma in the EMR was $0.1 \%$ (95\% UI $0.1-0.1 \%)$ in males and $0.2 \%$ (95\% UI $0.1-0.2 \%)$ in females in 1990 , and $0.1 \%$ (95\% UI $0.1-0.1 \%$ ) in males and $0.2 \%$ (95\% UI $0.1-0.2 \%$ ) in females in 2015 (ESM_4).

In 1990, glaucoma accounted for 10.7 age-standardized YLDs (95\% UI 7.3-14.8) per 100,000 for males and 15.4 age-standardized YLDs (95\% UI 10.5-21.1) per 100,000 for females in the EMR. The rate of age-standardized YLDs for glaucoma in 2015 was 11.5 (95\% UI 7.9-16.1) per 100,000 for males and 16.7 (95\% UI 11.6-23.1) per 100,000 females in this region (ESM_4). YLDs attributed to glaucoma were highest in Oman and lowest in Pakistan in 1990, and highest in Egypt and lowest in Lebanon in 2015.

\section{Macular degeneration}

The age-standardized prevalence of macular degeneration in the EMR was $0.1 \%$ (95\% UI $0.1-0.1 \%$ ) among males and $0.1 \%$ (95\% UI $0.1-0.1 \%$ ) among females in 1990. In 2015 , the corresponding values were $0.1 \%(0.1-0.1 \%)$ in males and $0.1 \%(95 \%$ UI $0.1-0.1 \%)$ in females in 2015 (ESM_5). Among the 22 countries, Kuwait had the highest and Afghanistan had the lowest prevalence of macular degeneration in 1990. However, macular degeneration was most prevalent in Oman and least prevalent in Somalia in 2015.

The age-standardized YLDs rate associated with macular degeneration in the EMR was 6.0 (95\% UI 4.1-8.3) per 100,000 for males and 6.5 (95\% UI 4.5-9.1) per 100,000 for females in 1990, increasing to 8.1 (95\% UI
5.6-11.2) per 100,000 males and 9.0 (95\% UI 6.2-12.6) per 100,000 females in 2015 (ESM_5). In both 1990 and 2015, Oman had the highest and Somalia had the lowest YLDs attributed to macular degeneration.

\section{Other causes of vision loss}

Saudi Arabia had the highest prevalence of other causes of vision loss, and Somalia had the lowest. In 1990, the agestandardized prevalence of other causes of vision loss was $0.4 \%$ (95\% UI $0.4-0.4 \%$ ) in males and $0.5 \%$ (95\% UI $0.4-0.5 \%)$ in females. Corresponding values were $0.5 \%$ (95\% UI $0.4-0.5 \%)$ in males and $0.5 \%$ (95\% UI $0.4-0.5 \%)$ in females in 2015 (ESM_6).

Oman had the largest burden of age-standardized YLDs rate for other causes of vision loss and Lebanon had the smallest in both 1990 and 2015. Age-standardized YLDs were 34.9 (95\% UI 24.7-47.6) per 100,000 males and 35.5 (95\% UI 25.0-47.8) per 100,000 females in 1990, and 37.7 (95\% UI 26.8-51.0) per 100,000 males and 38.6 (95\% UI 27.2-51.8) per 100,000 females in 2015 (ESM_6).

\section{Age-specific prevalence of the leading causes of vision loss}

The main causes of vision loss, including cataract, glaucoma, macular degeneration, and the category "other causes of vision loss" were most prevalent in the population aged 80 and older in both 1990 and 2015. The highest prevalence of refraction and accommodation disorders was noted among people aged 70-74 years in 1990 and 2015 (Fig. 3). 

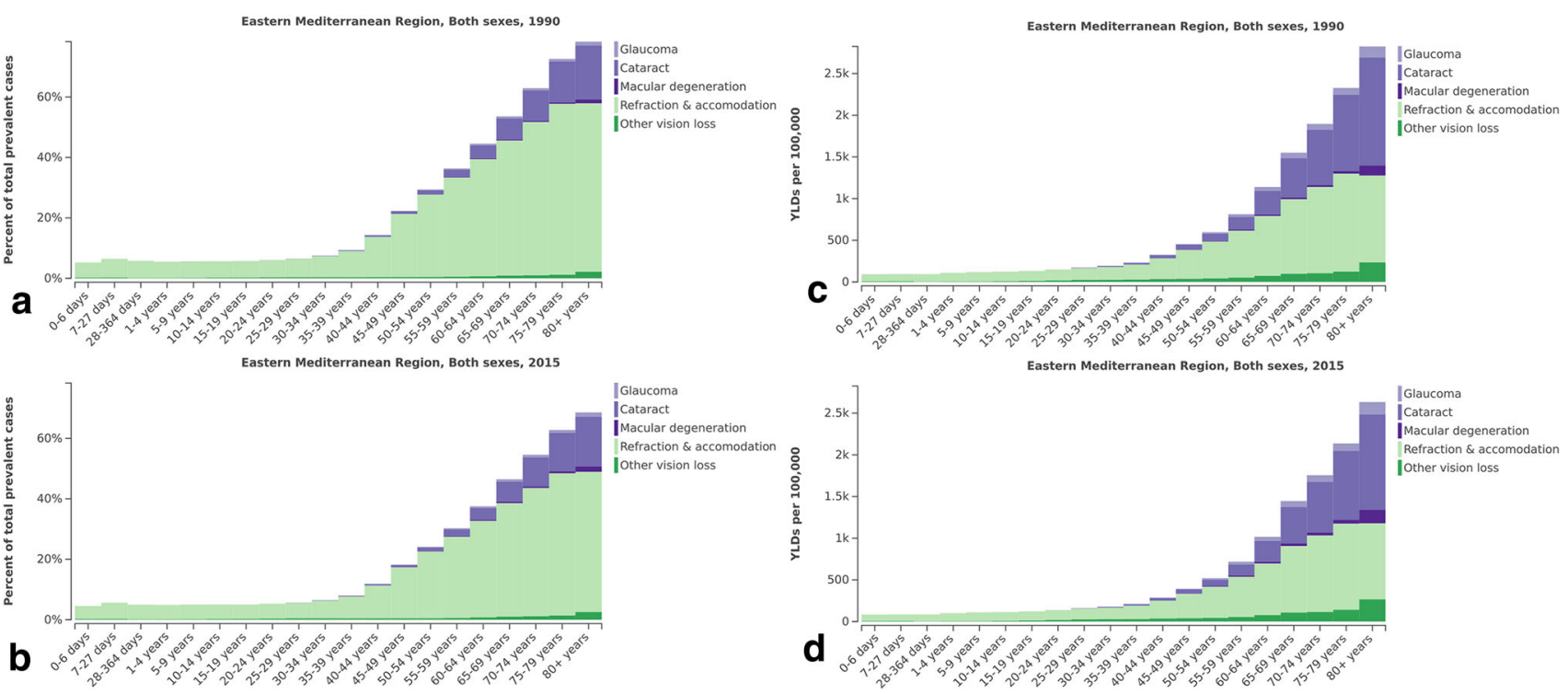

Fig. 3 Age-specific prevalence (a, b) and years lived with disability (YLDs) rate (c, d) for causes of vision loss in Eastern Mediterranean Region at two time points (1990 and 2015) (Global Burden of Disease Study 2015, Eastern Mediterranean Region, 1990, 2015)

\section{Leading causes of age-specific YLDs rate for vision} loss

Figure 3 shows the highest YLDs rates for cataract, glaucoma, macular degeneration, and other causes of vision loss in the population aged 80 or older in 1990 and 2015. The highest rate of YLDs for refraction and accommodation disorders was demonstrated in the age group 70-74 years in 1990 and 2015 (Fig. 3).

\section{Association of SDI and changes in age-standardized prevalence and YLDs rate of vision loss}

For each 0.1 unit increase in SDI, the age-standardized prevalence of vision loss due to all causes showed a $1.5 \%$ reduction using a multilevel linear model $(p<0.001)$. Corresponding values for each cause are presented in Table 2 and Fig. 4.

For each 0.1 unit increase in SDI, a 23.9 per 100,000 population reduction in the age-standardized YLDs rate for vision loss due to all causes was noted using a multilevel linear model $(p<0.001)$. Cause-specific YLDs are shown in Table 2 and Fig. 4.

\section{Ratio of observed-to-expected prevalence and YLDs of vision loss based on SDI}

ESM_7 shows that United Arab Emirates had the highest and Syria had the lowest observed-to-expected ratio $(\mathrm{O} / \mathrm{E})$ for prevalence of vision loss due to all causes based on SDI. The highest O/E YLDs ratio was noted in the Egypt, whereas Lebanon had the lowest ratio (ESM_8).

\section{Discussion}

This is the first report on the burden of vision loss in the EMR countries during 1990-2015 (GBD 2015). Our findings indicated a decline in the age-standardized and agespecific prevalence and YLDs rate of vision loss from 1990 to 2015. However, vision loss still presents a burden in the region and needs to be addressed in health policies.

Our findings on trends were compatible with the results of the Khairallah et al. study, which showed a descending trend in age-standardized prevalence of blindness and moderate and severe VI in the Middle East and North Africa from 1990 to 2010 (Khairallah et al. 2014). Despite a declining trend in age-standardized prevalence and YLDs rate of vision loss over the past 25 years in the EMR, a wide disparity among the 22 countries in this region was demonstrated in terms of vision loss prevalence and YLDs rate. This can be explained by the difference in socio-demographic conditions and capacities of health systems in these countries (Mandil et al. 2013; Mokdad et al. 2014). On the other hand, in recent years, a number of EMR countries have been involved in conflicts and wars, resulting in limited health resources (Mokdad et al. 2016). Unfavorable and unstable socioeconomic conditions can also lead to a lack of strategic plans and operational programs for the prevention and treatment of VI and blindness.

The EMR had a higher age-standardized prevalence and YLDs rate of vision loss compared to the global rate and ranked third following the Southeast Asia and Africa regions in 2015. Stevens et al. reported a greater than $4 \%$ prevalence of blindness in older adults in Western subSaharan Africa, Eastern sub-Saharan Africa, the EMR, and 
Table 2 Association of Sociodemographic Index and changes in age-standardized prevalence and years lived with disability rate of vision loss from 1990 to 2015 in the Eastern

Mediterranean Region (Global Burden of Disease Study 2015, Eastern Mediterranean Region, 1990-2015)
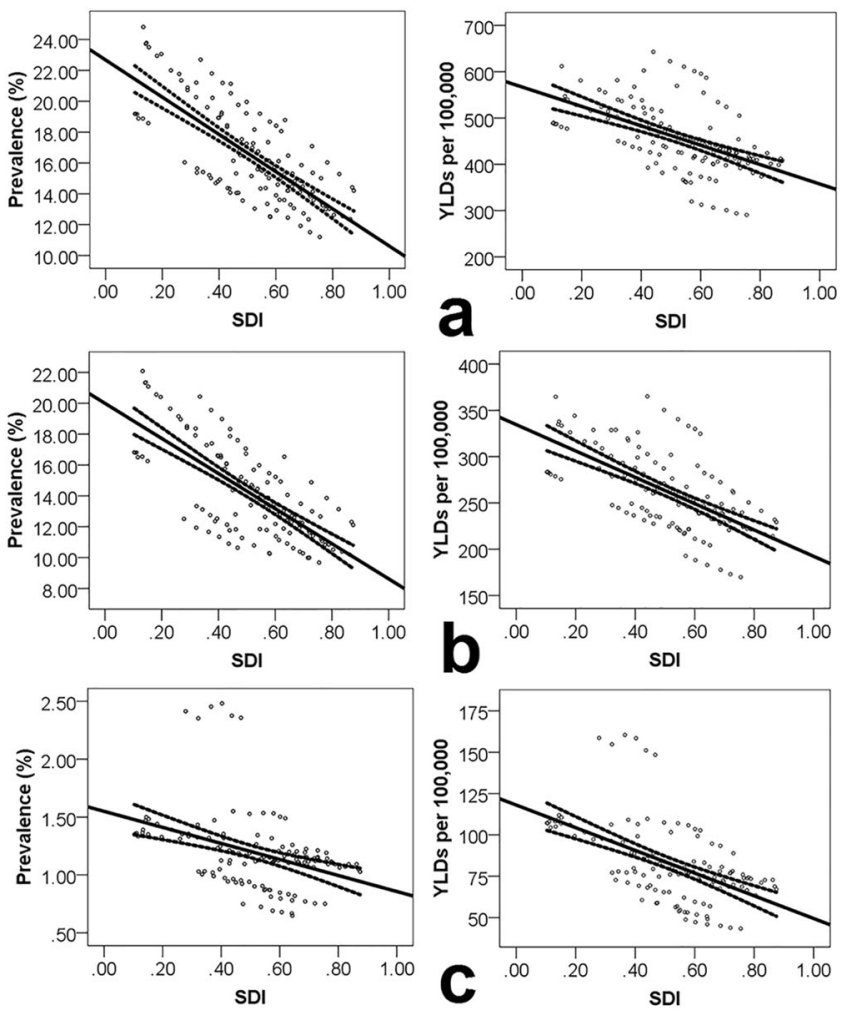

Fig. 4 Association of Socio-demographic Index (SDI) and changes in age-standardized prevalence and years lived with disability (YLDs) rate of all-cause vision loss (a), refraction and accommodation

South Asia in 2010 (Stevens et al. 2013). However, the highest reduction was observed in this region from 1990 to 2015 compared to all six world regions.

With regard to gender disparity, females were more affected by vision loss in the EMR, consistent with the global findings of the GBD 2015 study and other studies
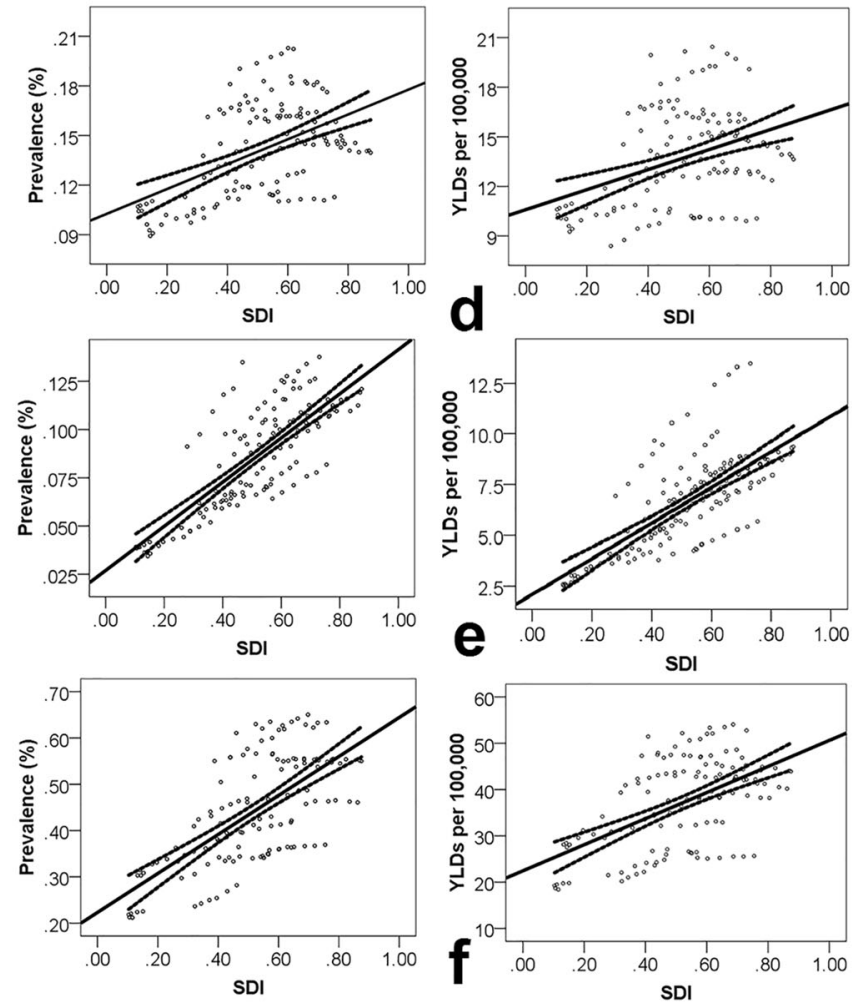

disorders (b), cataract (c), glaucoma (d), macular degeneration (e), and other causes of vision loss (f) (Global Burden of Disease Study 2015, Eastern Mediterranean Region, 2015)

reporting on the EMR (Abou-Gareeb et al. 2001; GBD 2015; Hashemi et al. 2012; Jadoon et al. 2006; Khairallah et al. 2014; Rajavi et al. 2016; Stevens et al. 2013; WHO 2007). The gender disparity might be due to allocation of less family financial resources, resulting in limited access to eye care services for females (Hashemi et al. 2012; 
Stevens et al. 2013). This inequality may be attributed to cultural backgrounds which could be overcome by promoting awareness and education in affected societies.

Our results follow previous global and regional studies that reported refraction and accommodation disorders, cataract, glaucoma, and macular degeneration as the main causes of vision loss (GBD 2015; Khairallah et al. 2014; Katibeh et al. 2017a, b; Köberlein et al. 2013; Naidoo et al. 2014; Keeffe et al. 2014). Among these, refraction and accommodation disorders remained the most prevalent in the EMR from 1990 to 2015. A similar global-scale finding was reported in the GBD 2015 study and WHO global data 2010 (GBD 2015; Pascolini and Mariotti 2012; Bourne et al. 2013; Resnikoff et al. 2008; Naidoo et al. 2016). However, a decreasing trend in age-standardized prevalence and YLDs rate due to refraction and accommodation disorders was observed in this region. There was a $1.46 \%$ reduction in age-standardized prevalence and 17.89 per 100,000 population reduction in YLDs from refraction and accommodation disorders per 0.1 unit improvement in SDI score in the current study. Refraction and accommodation disorders had a considerable impact on the socioeconomic condition of the affected persons and their families via limiting educational and employment opportunities, resulting in productivity loss (Naidoo and Jaggernath 2012; Smith et al. 2009). A large amount of vision loss can be potentially prevented and cured through developing and implementing national screening programs and cost-effective interventions (WHO 2013).

Cataract was the second-ranked cause of vision loss in the EMR in both 1990 and 2015, with female predominance that was compatible with global findings from the GBD 2015 study (GBD 2015). The global WHO report also indicated that cataracts accounted for $33 \%$ of VI and 51\% of blindness in 2010 (Pascolini and Mariotti 2012). Considering the geographic location of EMR countries, ultraviolet radiation may play a role in the high prevalence of cataract in this region (McCarty and Taylor 2002). Cataract can be treated simply with a timely and costeffective intervention which would result in favorable visual outcomes; therefore it is categorized as a preventable cause of blindness (Pascolini and Mariotti 2012; WHO 2013). We found a significant correlation between the improvement of SDI score (by 0.1 unit) and the $0.04 \%$ reduction in age-standardized prevalence and 4.41 per 100,000 population reduction in the rate of YLDs from vision loss due to cataract in the EMR, which was in line with the Mundy et al. study. They reviewed the literature to report the association of cataract care with socioeconomic parameters in both developed and developing countries. These parameters can lead to limited access to primary eye care services. Promotion of education can improve the acceptability of these resources in low socioeconomic areas (Mundy et al. 2016).

Glaucoma was the third-leading cause of vision loss in the EMR. A slightly ascending trend of age-standardized prevalence and the rate of YLDs from glaucoma were observed over our study period. This finding follows the global results in the GBD 2015 study (GBD 2015). A metaanalysis by Bourne et al. showed an increased percentage of blindness due to glaucoma from 1990 to 2010 globally, with no significant difference between regions (Bourne et al. 2016). We also found that the slightly increasing age-standardized YLDs rate of glaucoma (0.25 per 100,000 person) was associated with the improved SDI score (by 0.1 unit). Given the increasing trend of vision loss due to glaucoma, a number of issues should be considered for public health planning. First, glaucoma and macular degeneration are responsible for the majority of irreversible blindness in the world. One out of 15 cases of blindness and one out of 45 cases of VI were due to glaucoma by 2010 (Bourne et al. 2016). Early diagnosis and proper medical and surgical management can prevent blindness due to glaucoma. Secondly, the Bourne et al. study also revealed that regions with younger populations had lower percentages of blindness due to glaucoma compared to high-income regions with older populations (Bourne et al. 2016). Tham et al. demonstrated the highest prevalence of glaucoma in Africa (primary openangle glaucoma $4.20 \%$ ) and Asia (primary angle-closure glaucoma 1.20\%) in 2013 (Tham et al. 2014). Therefore, considering increasing life expectancy, the prevalence of VI due to glaucoma is expected to increase in the EMR in the future. Clinical and targeted screening would be appropriate approaches for preventing vision loss due to glaucoma (Mohammadi et al. 2014).

Macular degeneration was the fourth-leading cause of vision loss in terms of prevalence and YLDs rate in the EMR. An increasing trend was observed in age-standardized prevalence and rate of YLDs due to macular degeneration in both sexes. Our finding is consistent with the global results from GBD 2015 and the meta-analysis from 1990 to 2010 (GBD 2015; Jonas et al. 2014). This metaanalysis demonstrated a lower prevalence of macular degeneration in regions with younger populations in comparison with high-income regions (Jonas et al. 2014). Our study also showed that the increase in age-standardized prevalence $(0.01 \%)$ and YLDs rate $(0.9$ per 100,000 person) of macular degeneration from 1990 to 2015 was associated with the improvement of SDI score (by 0.1 unit). With regard to the aging population and availability of effective interventions, especially intravitreal anti-vascular endothelial growth factor drugs, macular degeneration may be recognized as an important public eye health issue for future planning (Jonas et al. 2014). 
Our study has a few limitations. Vision loss due to diabetes mellitus is considered part of the diabetes burden and is not included in our study (Moradi-Lakeh et al. 2017). Considering the high prevalence of diabetes in EMR, the burden of VI may be higher than what is estimated in this study (Katibeh et al. 2017a, b; Khandekar 2012; WHO 2016). Some of the countries in the region do not have appropriate data on the epidemiology of low vision or have used nonstandard methods for measuring and reporting vision loss. We used the GBD general methodology to produce more accurate estimates using different study-level or country-level covariates.

\section{Conclusions}

The current study provides an up-to-date estimation based on the GBD 2015 study, demonstrating a high prevalence and high rate of YLDs due to vision loss with a decreasing trend in the EMR. Our findings call for developing and implementing programs to manage refraction and accommodation disorders and cataract due to their large burden. There is a need for balance between prevention and treatment programs to reduce the burden of vision loss in the region. This can be achieved by developing and implementing a national operational program and involving all related stakeholders. Education campaigns might be useful to promote public awareness.

GBD 2015 Eastern Mediterranean Region Vision Loss Collaborators: Ali $\mathrm{H}$. Mokdad, $\mathrm{PhD}$ (corresponding author), Institute for Health Metrics and Evaluation, University of Washington, Seattle, Washington, United States. Sare Safi, MS, Ophthalmic Epidemiology Research Center, Shahid Beheshti University of Medical Sciences, Tehran, Iran. Hamid Ahmadieh, MD, Opthalmic Research Center, Shahid Beheshti University of Medical Sciences, Tehran, Iran. Marzieh Katibeh, MD, Center for Global Health, Aarhus University, Aarhus, Denmark. Mehdi Yaseri, PhD, Department of Epidemiology and Biostatistics, School of Public Health, Tehran University of Medical Sciences, Tehran, Iran. Alireza Ramezani, MD, Ophthalmic Epidemiology Research Center, Shahid Beheshti University of Medical Sciences, Tehran, Iran. Saeid Shahraz, MD, Tufts Medical Center, Boston, MA, United States. Maziar Moradi-Lakeh, MD, Department of Community Medicine, Preventive Medicine and Public Health Research Center, Gastrointestinal and Liver Disease Research Center (GILDRC), Iran University of Medical Sciences, Tehran, Iran. Ibrahim Khalil, PhMD, Institute for Health Metrics and Evaluation, University of Washington, Seattle, Washington, United States. Charbel El Bcheraoui, PhD, Institute for Health Metrics and Evaluation, University of Washington, Seattle, Washington, United States. Michael Collison, BS, Institute for Health Metrics and Evaluation, University of Washington, Seattle, Washington, United States. Adrienne Chew, ND, Institute for Health Metrics and Evaluation, University of Washington, Seattle, Washington, United States. Farah Daoud, BA/BS, Institute for Health Metrics and Evaluation, University of Washington, Seattle, Washington, United States. Kristopher J. Krohn, BA, Institute for Health Metrics and Evaluation, University of Washington, Seattle, Washington, United States. Zane Rankin, BA/ BS, Institute for Health Metrics and Evaluation, University of Washington, Seattle, Washington, United States. Ashkan Afshin, MD,
Institute for Health Metrics and Evaluation, University of Washington, Seattle, Washington, United States. Nicholas J. Kassebaum, MD, Institute for Health Metrics and Evaluation, University of Washington, Seattle, Washington, United States; Department of Anesthesiology and Pain Medicine, Seattle Children's Hospital, United States. Helen E. Olsen, MA, Institute for Health Metrics and Evaluation, University of Washington, Seattle, Washington, United States. Jeffrey D. Stanaway, PhD, Institute for Health Metrics and Evaluation, University of Washington, Seattle, Washington, United States. Haidong Wang, PhD, Institute for Health Metrics and Evaluation, University of Washington, Seattle, Washington, United States. Katie Wilson, MLIS, Institute for Health Metrics and Evaluation, University of Washington, Seattle, Washington, United States. Gebre Yitayih, MS, Mekelle University, Mekelle, Ethiopia. Ayman AlEyadhy, MD, King Saud University, Riyadh, Saudi Arabia. Khurshid Alam, PhD, Murdoch Childrens Research Institute, The University of Melbourne, Parkville, Victoria, Australia; The University of Melbourne, Melbourne, VIC, Australia. Deena Alasfoor, MSc, Ministry of Health, Al Khuwair, Muscat, Oman. Reza Alizadeh-Navaei, PhD, Gastrointestinal Cancer Research Center, Mazandaran University of Medical Sciences, Sari, Iran. Rajaa Al-Raddadi, PhD, Joint Program of Family and Community Medicine, Jeddah, Saudi Arabia. Ubai Alsharif, MPH, Charité Universitätsmedizin, Berlin, Germany. Khalid A. Altirkawi, MD, King Saud University, Riyadh, Saudi Arabia. Nahla Anber, PhD, Mansoura University, Mansoura, Egypt. Hossein Ansari, PhD, Health Promotion Research Center, Department of Epidemiology and Biostatistics, Zahedan University of Medical Sciences, Zahedan, Iran. Palwasha Anwari, MD, Self-employed, Kabul, Afghanistan. Hamid Asayesh, PhD, Department of Medical Emergency, School of Paramedic, Qom, Iran; University of Medical Sciences, Qom, Iran; Solomon Weldegebreal Asgedom, PhD, Mekelle University, Mekelle, Ethiopia. Tesfay Mehari Atey, MS, Mekelle University, Mekelle, Ethiopia. Umar Bacha, PhD, School of Health Sciences, University of Management and Technology, Lahore, Pakistan. Aleksandra Barac, PhD, Faculty of Medicine, University of Belgrade, Belgrade, Serbia. Neeraj Bedi, MD, College of Public Health and Tropical Medicine, Jazan, Saudi Arabia. Zahid A. Butt, $\mathrm{PhD}$, Al Shifa Trust Eye Hospital, Rawalpindi, Pakistan. Abdulaal A. Chitheer, MD, Ministry of Health, Baghdad, Iraq. Shirin Djalalinia, $\mathrm{PhD}$, Undersecretary for Research and Technology, Ministry of Health and Medical Education, Tehran, Iran. Huyen Do Phuc, MSc, Institute for Global Health Innovations, Duy Tan University, Da Nang, Vietnam. Babak Eshrati, PhD, Ministry of Health and Medical Education, Tehran, Iran; Arak University of Medical Sciences, Arak, Iran. Maryam S. Farvid, PhD, Department of Nutrition, Harvard T. H. Chan School of Public Health, Harvard University, Boston, MA, United States; Harvard/MGH Center on Genomics, Vulnerable Populations, and Health Disparities, Mongan Institute for Health Policy, Massachusetts General Hospital, Boston, MA, United States. Farshad Farzadfar, MD, Non-communicable Diseases Research Center, Tehran University of Medical Sciences, Tehran, Iran. Seyed-Mohammad, Fereshtehnejad, PhD, Department of Neurobiology, Care Sciences and Society (NVS), Karolinska Institutet, Stockholm, Sweden. Florian Fischer, PhD, School of Public Health, Bielefeld University, Bielefeld, Germany. Tsegaye Tewelde Gebrehiwot, MPH, Jimma University, Jimma, Ethiopia. Randah Ribhi Hamadeh, Arabian Gulf University, Manama, Bahrain. Samer Hamidi, DPhil, Hamdan Bin Mohammed Smart University, Dubai, United Arab Emirates. Tarig B. Higazi, $\mathrm{PhD}$, The Ohio University, Zanesville, Ohio, United States. Mohamed Hsairi, MD, Department of Epidemiology, Salah Azaiz Institute, Tunis, Tunisia. Aida Jimenez-Corona, $\mathrm{PhD}$, Department of Ocular Epidemiology and Visual Health, Institute of Ophthalmology Conde de Valencia, Mexico City, Mexico; General Directorate of Epidemiology, Ministry of Health, Mexico City, Mexico. Denny John, MPH, International Center for Research on Women, New Delhi, India. Jost B. Jonas, MD, Department of Ophthalmology, Medical 
Faculty Mannheim, Ruprecht-Karls-University Heidelberg, Mannheim, Germany. Amir Kasaeian, PhD, Hematology-Oncology and Stem Cell Transplantation Research Center, Tehran, Iran; University of Medical Sciences, Tehran, Iran; Endocrinology and Metabolism Population Sciences Institute, Tehran University of Medical Sciences, Tehran, Iran. Yousef Saleh, ScD, Department of Community Medicine, Public Health and Family Medicine, Jordan University of Science and Technology, Irbid, Jordan. Ejaz Ahmad Khan, MD, Health Services Academy, Islamabad, Pakistan. Heidi J. Larson, PhD, Department of Infectious Disease Epidemiology, London School of Hygiene and Tropical Medicine, London, United Kingdom; Institute for Health Metrics and Evaluation, University of Washington, Seattle, Washington, United States. Asma Abdul Latif, PhD, Department of Zoology, Lahore College for Women University, Lahore, Pakistan. Raimundas Lunevicius, PhD, Aintree University Hospital National Health Service Foundation Trust, Liverpool, United Kingdom; School of Medicine, University of Liverpool, Liverpool, United Kingdom. Hassan Magdy Abd El Razek, MBBCH, Mansoura Faculty of Medicine, Mansoura, Egypt. Mohammed Magdy Abd El Razek, MBBCH, Aswan University Hospital, Aswan Faculty of Medicine, Aswan, Egypt. Azeem Majeed, MD, Department of Primary Care and Public Health, Imperial College London, London, United Kingdom. Reza Malekzadeh, MD, Digestive Diseases Research Institute, Tehran University of Medical Sciences, Tehran, Iran. Colm McAlinden, PhD, University Hospitals Bristol NHS Foundation Trust, Bristol, United Kingdom; Public Health Wales, Swansea, United Kingdom. Ziad A. Memish, MD, Saudi Ministry of Health, Riyadh, Saudi Arabia; College of Medicine, Alfaisal University, Riyadh, Saudi Arabia. Ted R. Miller, $\mathrm{PhD}$, Pacific Institute for Research and Evaluation, Calverton, MD, United States; Centre for Population Health, Curtin University, Perth, WA, Australia. Seyed-Farzad Mohammadi, MD, Translational Ophthalmology Research Center, Farabi Eye Hospital, Tehran University of Medical Sciences, Tehran, Iran. Vinay Nangia, MD, Suraj Eye Institute, Nagpur, India. Cuong Tat Nguyen, MSc, Institute for Global Health Innovations, Duy Tan University, Da Nang, Vietnam. Quyen Le Nguyen, MD, Institute for Global Health Innovations, Duy Tan University, Da Nang, Vietnam. Felix Akpojene Ogbo, MPH, Centre for Health Research, Western Sydney University, Sydney, New South Wales, Australia. Farshad Pourmalek, PhD, University of British Columbia, Vancouver, British Columbia, Canada. Mostafa Qorbani, PhD, Non-communicable Diseases Research Center, Alborz University of Medical Sciences, Karaj, Iran. Anwar Rafay, MS, Contech International Health Consultants, Lahore, Pakistan; Contech School of Public Health, Lahore, Pakistan. Vafa Rahimi-Movaghar, MD, Sina Trauma and Surgery Research Center, Tehran University of Medical Sciences, Tehran, Iran. Rajesh Kumar Rai, MPH, Society for Health and Demographic Surveillance, Suri, India. Saleem M. Rana, $\mathrm{PhD}$, Contech School of Public Health, Lahore, Pakistan; Contech International Health Consultants, Lahore, Pakistan. David Laith Rawaf, MD, WHO Collaborating Centre, Imperial College London, London, United Kingdom; North Hampshire Hospitals, Basingstroke, United Kingdom. Salman Rawaf, MD, Imperial College London, London, United Kingdom. Andre M.N. Renzaho, PhD, Western Sydney University, Penrith, NSW, Australia. Satar Rezaei, PhD, School of Public Health, Kermanshah University of Medical Sciences, Kermanshah, Iran. Gholamreza Roshandel, PhD, Golestan Research Center of Gastroenterology and Hepatology, Golestan University of Medical Sciences, Gorgan, Iran; Digestive Diseases Research Institute, Tehran University of Medical Sciences, Tehran, Iran. Mahdi Safdarian, MD, Sina Trauma and Surgery Research Center, Tehran University of Medical Sciences, Tehran, Iran. Saeid Safiri, PhD, Managerial Epidemiology Research Center, Department of Public Health, School of Nursing and Midwifery, Maragheh University of Medical Sciences, Maragheh, Iran. Payman Salamati,
MD, Sina Trauma and Surgery Research Center, Tehran University of Medical Sciences, Tehran University of Medical Sciences, Tehran, Iran. Abdallah M. Samy, PhD, Ain Shams University, Cairo, Egypt. Benn Sartorius, PhD, Public Health Medicine, School of Nursing and Public Health, University of KwaZulu-Natal, Durban, South Africa; UKZN Gastrointestinal Cancer Research Centre, South African Medical Research Council (SAMRC), Durban, South Africa. Sadaf G. Sepanlou, PhD, Digestive Diseases Research Institute, Tehran University of Medical Sciences, Tehran, Iran. Masood Ali Shaikh, MD, Independent Consultant, Karachi, Pakistan. Eirini Skiadaresi, MD, Hywel Dda University Health Board, Carmarthen, United Kingdom. Badr H. A. Sobaih, MD, King Saud University, Riyadh, Saudi Arabia. Rizwan Suliankatchi Abdulkader, MD, Ministry of Health, Kingdom of Saudi Arabia, Riyadh, Saudi Arabia. Hugh R. Taylor, AC, University of Melbourne, Carlton, Victoria, Australia. Arash Tehrani-Banihashemi, PhD, Preventive Medicine and Public Health Research Center, Iran University of Medical Sciences, Tehran, Iran. Mohamad-Hani Temsah, MD, King Saud University, Riyadh, Saudi Arabia. Roman Topor-Madry, PhD, Institute of Public Health, Faculty of Health Sciences, Jagiellonian University Medical College, Krakow, Poland; Faculty of Health Sciences, Wroclaw Medical University, Wroclaw, Poland. Bach Xuan, PhD, Johns Hopkins University, Baltimore, Maryland, United States; Hanoi Medical University, Hanoi, Vietnam. Miltiadis Tsilimbaris, PhD, Department of Medicine, University of Crete, Heraklion, Greece. Kingsley Nnanna Ukwaja, MD, Department of Internal Medicine, Federal Teaching Hospital, Abakaliki, Ebonyi State, Nigeria. Olalekan A. Uthman, PhD, Warwick Medical School, University of Warwick, Coventry, United Kingdom. Tolassa Wakayo, MS, Jimma University, Jimma, Ethiopia. Naohiro Yonemoto, MPH, Department of Biostatistics, School of Public Health, Kyoto University, Kyoto, Japan. Mustafa Z. Younis, PH, Jackson State University, Jackson, MS, United States. Maysaa El Sayed Zaki, PhD, Faculty of Medicine, Mansoura University, Mansoura, Egypt. Aisha O. Jumaan, PhD, Independent Consultant, Seattle, Washington, United States. Theo Vos, $\mathrm{PhD}$, Institute for Health Metrics and Evaluation, University of Washington, Seattle, Washington, United States. Simon I. Hay, DSc, Oxford Big Data Institute, Li Ka Shing Centre for Health Information and Discovery, University of Oxford, Oxford, London, United Kingdom; Institute for Health Metrics and Evaluation, University of Washington, Seattle, Washington, United States. Mohsen Naghavi, $\mathrm{PhD}$, Institute for Health Metrics and Evaluation, University of Washington, Seattle, Washington, United States. Christopher J.L. Murray, DPhil, Institute for Health Metrics and Evaluation, University of Washington, Seattle, Washington, United States.

\section{Compliance with ethical standards}

Ethical approval This manuscript reflects original work that has not previously been published in whole or in part and is not under consideration elsewhere. All authors have read the manuscript and have agreed that the work is ready for submission and accept responsibility for its contents. The authors of this paper have complied with all ethical standards and do not have any conflicts of interest to disclose at the time of submission. The funding source played no role in the design of the study, the analysis and interpretation of data, and the writing of the paper. The study did not involve human participants and/or animals; therefore, no informed consent was needed.

Conflict of interest The authors declare that they have no conflicts of interest.

Funding This research was funded by the Bill \& Melinda Gates Foundation. 
Open Access This article is distributed under the terms of the Creative Commons Attribution 4.0 International License (http://crea tivecommons.org/licenses/by/4.0/), which permits unrestricted use, distribution, and reproduction in any medium, provided you give appropriate credit to the original author(s) and the source, provide a link to the Creative Commons license, and indicate if changes were made.

\section{References}

Abou-Gareeb I, Lewallen S, Bassett K et al (2001) Gender and blindness: a meta-analysis of population-based prevalence surveys. Ophthalmic Epidemiol 8:39-56

Bates D, Mächler M, Bolker B, Walker S (2015) Fitting linear mixedeffects models using lme4. J Stat Softw 67:1-48. doi:10.18637/ jss.v067.i01

Bourne RR, Stevens GA, White RA, Vision Loss Expert Group et al (2013) Causes of vision loss worldwide, 1990-2010: a systematic analysis (2013). Lancet Glob Health 1:339-349. doi:10. 1016/S2214-109X(13)70113-X

Bourne RR, Taylor HR, Flaxman SR, Vision Loss Expert Group of the Global Burden of Disease Study et al (2016) Number of people blind or visually impaired by glaucoma worldwide and in world regions 1990-2010: a meta-analysis. PLoS One 11:2229. doi:10.1371/journal.pone.0162229

Congdon NG, Friedman DS, Lietman T (2003) Important causes of visual impairment in the world today. JAMA 290:2057-2060. doi:10.1001/jama.290.15.2057

GBD 2015 Disease and Injury Incidence and Prevalence Collaborators (2015) Global, regional, and national incidence, prevalence, and years lived with disability for 310 diseases and injuries, 1990-2015: a systematic analysis for the Global Burden of Disease Study. Lancet 388(10053):1545-1602. doi:10.1016/ S0140-6736(16)31678-6

Hashemi H, Khabazkhoob M, Emamian MH et al (2012) Visual impairment in the 40- to 64-year-old population of Shahroud, Iran. Eye (Lond) 26:1071-1077. doi:10.1038/eye.2012.94

Jadoon MZ, Dineen B, Bourne RR et al (2006) Prevalence of blindness and visual impairment in Pakistan: the Pakistan National Blindness and Visual Impairment Survey. Invest Ophthalmol Vis Sci 47:4749-4755. doi:10.1167/iovs.06-0374

Jonas JB, Bourne RR, White RA, Vision Loss Expert Group of the Global Burden of Disease Study et al (2014) Visual impairment and blindness due to macular diseases globally: a systematic review and meta-analysis. Am J Ophthalmol 158:808-815. doi:10.1016/j.ajo.2014.06.012

Katibeh M, Behboudi H, Moradian S et al (2017a) Rapid assessment of avoidable blindness and diabetic retinopathy in Gilan province, Iran. Ophthalmic Epidemiol 19:1-7. doi:10.1080/ 09286586.2017

Katibeh M, Kalantarion M, Mariotti SP et al (2017b) A stakeholder perspective on diabetes mellitus and diabetic retinopathy care in Iran; a qualitative study. Arch Iran Med 20:288-294. doi: 0172005/AIM.006

Keeffe J, Taylor HR, Fotis K, Vision Loss Expert Group of the Global Burden of Disease Study et al (2014) Prevalence and causes of vision loss in Southeast Asia and Oceania: 1990-2010. Br J Ophthalmol 98:586-591. doi:10.1136/bjophthalmol-2013-304050

Khairallah M, Kahloun R, Flaxman SR, Vision Loss Expert Group et al (2014) Prevalence and causes of vision loss in North Africa and the Middle East: 1990-2010. Br J Ophthalmol 98:605-611. doi:10.1136/bjophthalmol-2013-304068
Khandekar R (2012) Screening and public health strategies for diabetic retinopathy in the Eastern Mediterranean region. Middle East Afr J Ophthalmol 19:178-184. doi:10.4103/0974-9233.95245

Köberlein J, Beifus K, Schaffert C et al (2013) The economic burden of visual impairment and blindness: a systematic review. BMJ Open 3:e003471. doi:10.1136/bmjopen-2013-003471

Mandil A, Chaaya M, Saab D (2013) Health status, epidemiological profile and prospects: Eastern Mediterranean region. Int $\mathbf{J}$ Epidemiol 42:616-626. doi:10.1093/ije/dyt026

McCarty CA, Taylor HR (2002) A review of the epidemiologic evidence linking ultraviolet radiation and cataracts. Dev Ophthalmol 35:21-31

McCarty CA, Nanjan MB, Taylor HR (2001) Vision impairment predicts 5 year mortality. Br J Ophthalmol 85:322-326

Mohammadi S-F, Saeedi-Anari G, Alinia C, Ashrafi E et al (2014) Is screening for glaucoma necessary? A policy guide and analysis. J Ophthalmic Vis Res 9:3-6

Mokdad AH, Jaber S, Aziz MIA et al (2014) The state of health in the Arab world, 1990-2010: an analysis of the burden of diseases, injuries, and risk factors. Lancet 383:309-320. doi:10.1016/ S0140-6736(13)62189-3

Mokdad AH, Forouzanfar MH, Daoud F et al (2016) Health in times of uncertainty in the Eastern Mediterranean region, 1990-2013: a systematic analysis for the Global Burden of Disease Study 2013. Lancet Glob Health 4:e704-e713. doi:10.1016/S2214109X(16)30168-1

Moradi-Lakeh M, Forouzanfar MH, Bcheraoui CE, et al (2017) High Fasting Plasma Glucose, Diabetes, and Its Risk Factors in the Eastern Mediterranean Region, 1990-2013: Findings From the Global Burden of Disease Study 2013. Diabetes Care 40:22-29. doi: $10.2337 / \mathrm{dc} 16-1075$

Mundy KM, Nichols E, Lindsey J (2016) Socioeconomic disparities in cataract prevalence, characteristics, and management. Semin Ophthalmol 31:358-363. doi:10.3109/08820538.2016.1154178

Naidoo KS, Jaggernath J (2012) Uncorrected refractive errors. Indian J Ophthalmol 60:432-437. doi:10.4103/0301-4738

Naidoo K, Gichuhi S, Basáñez MG, Vision Loss Expert Group of the Global Burden of Disease Study et al (2014) Prevalence and causes of vision loss in sub-Saharan Africa: 1990-2010. Br J Ophthalmol 98:612-618. doi:10.1136/bjophthalmol-2013304081

Naidoo KS, Leasher J, Bourne RR, Vision Loss Expert Group of the Global Burden of Disease Study et al (2016) Global vision impairment and blindness due to uncorrected refractive error, 1990-2010. Optom Vis Sci 93:227-234. doi:10.1097/OPX. 0000000000000796

Pascolini D, Mariotti SP (2012) Global estimates of visual impairment: 2010. Br J Ophthalmol 96:614-618. doi:10.1136/bjophthal mol-2011-300539

Rajavi Z, Mokhtari S, Sabbaghi H et al (2016) Long-term visual outcome of congenital cataract at a tertiary referral center from 2004 to 2014. J Curr Ophthalmol 27:103-109. doi:10.1016/j. joco.2015.11.001

Resnikoff S, Pascolini D, Mariotti SP et al (2008) Global magnitude of visual impairment caused by uncorrected refractive errors in 2004. Bull World Health Organ 86:63-70

Smith TS, Frick KD, Holden BA et al (2009) Potential lost productivity resulting from the global burden of uncorrected refractive error. Bull World Health Organ 87:431-437

Stevens GA, White RA, Flaxman SR et al (2013) Global prevalence of vision impairment and blindness: magnitude and temporal trends, 1990-2010. Ophthalmology 120:2377-2384. doi:10. 1016/j.ophtha.2013.05.025

Tabbara KF (2001) Blindness in the eastern Mediterranean countries. Br J Ophthalmol 85:771-775. doi:10.1136/bjo.85.7.771 
Taylor HR, Katala S, Muñoz B, Turner V (1991) Increase in mortality associated with blindness in rural Africa. Bull World Health Organ 69:335-338

Taylor HR, Pezzullo ML, Keeffe JE (2006) The economic impact and cost of visual impairment in Australia. $\mathrm{Br} \mathrm{J}$ Ophthalmol 90:272-275. doi:10.1136/bjo.2005.080986

Tham YC, Li X, Wong TY et al (2014) Global prevalence of glaucoma and projections of glaucoma burden through 2040: a systematic review and meta-analysis. Ophthalmology 121:2081-2090. doi:10.1016/j.ophtha.2014.05.013

World Health Organization (2004) World health report 2004. Changing history. World Health Organization, Geneva. Table 3, p 130. http://www.who.int/whr/2004/en/report04_en.pdf. Accessed 2 Mar 2017
World Health Organization (2007) Global initiative for the elimination of avoidable blindness action plan 2006-2011. WHO Press, Geneva

World Health Organization (2013) Action plan for the prevention of avoidable blindness and visual impairment 2014-2019: towards universal eye health: a global action plan 2014-2019. http:// www.who.int/blindness/actionplan/en/index.html. Accessed 10 Sept 2016

World Health Organization (2016) Global report on diabetes. World Health Organization, Geneva. http://apps.who.int/iris/bitstream/ 10665/204871/1/9789241565257_eng.pdf. Accessed 1 Oct 2017

World Health Organization (2017) Eastern Mediterranean Region. http://www.emro.who.int/entity/about-us/index.html. Accessed 2 Mar 2017 